\title{
Construction and Performance Studies of Large Resistive Micromegas Quadruplets
}

\author{
A. Düdder, Tai-Hua Lin*, M. Schott, C.Valderanis \\ Johannes-Gutenberg-Universitaet Mainz (DE) \\ E-mail: tlinecern.ch \\ M. Bianco, ${ }^{a}$ H. Danielsson, ${ }^{a}$ J. Degrange,${ }^{a}$ R. De Oliveira, ${ }^{a}$ E. Farina,${ }^{a b}$ F. Kuger,${ }^{a c}$ \\ P. lengo, ${ }^{a}$ F. Perez Gomez, ${ }^{a}$ G. Sekhniaidze, ${ }^{d}$ O. Sidiropoulou, ${ }^{a c}$ M. Vergain, ${ }^{a}$ and \\ J. Wotschack ${ }^{a}$ \\ ${ }^{a}$ CERN PH Department, Geneve $(\mathrm{CH})$ \\ ${ }^{b}$ University of Pavia (IT) \\ ${ }^{c}$ Bayerische Julius Max. Universitaet Würzburg (DE) \\ ${ }^{d}$ INFN and University of Naples (IT)
}

\begin{abstract}
Two resistive Micromegas quadruplet detectors with trapezoidal shape and an area of $0.5 \mathrm{~m}^{2}$ were constructed, serving as prototypes for the future chambers of the ATLAS New Small Wheel (NSW) upgrade. Each quadruplet consists of two double-sided readout panels and three drift panels (one double face), equipped with a micromesh, the drift electrode and gas pipes. There are four detection layers each with an active area of $0.3 \mathrm{~m}^{2}$. Two layers have readout strips parallel to the base of the trapezoid while the other two have strips inclined by $\pm 1.5^{\circ}$ with respect to the first ones. In this paper we present the first results for the performance of the detectors, in test-beam measurement and cosmic ray tests.
\end{abstract}

The European Physical Society Conference on High Energy Physics

22-29 July 2015

Vienna, Austria

${ }^{*}$ Speaker. 


\section{Introduction}

MICRO Mesh GAseous Structures (Micromegas) are gaseous parallel plate detectors consisting of two asymmetric electric field regions, the drift and amplification region, separated by a thin metallic mesh. In the detector layout adopted by the ATLAS NSW upgrade, a layer of resistive strips is cladded by spattering on top of the readout strips for spark tolerance. This work summarizes the construction and first performance studies of two $0.5 \mathrm{~m}^{2}$ Micromegas quadruplets. These prototypes serve the purpose of evaluating the detector design and construction towards the production of the Micromegas detectors that will be installed in the ATLAS Small Wheels. Special focus is drawn on first performance studies based on test-beam and cosmic ray measurements.

\section{Detector Design}

A schematic drawing of the prototypes is shown in Fig. 1. Each quadruplet consists of three drift and two readout panels with trapezoidal shape. The readout panels are $20 \mathrm{~cm}$ longer than the drift panels, in the upper and lower base, in order to host the readout electronics. The readout panels are formed by two back-to-back FR4 printed circuit boards (PCB) with aluminum honeycomb in between and surrounded by aluminum profile as supporting structure. Each readout PCB hosts 1024 readout strips with a width of $300 \mu \mathrm{m}$ and a pitch of $415 \mu \mathrm{m}$. Two readout layers have strips parallel to the trapezoidal base for the reconstruction of the precision coordinate, while the other two have strips inclined by a $\pm 1.5^{\circ}$ with respect to other layers for the reconstruction of the precision and second coordinate [3]. This strip pattern allows for a precise measurement of the spatial resolution.

The drift panels have a similar structure as the readout panels but instead of the readout strips they are clad with a copper layer to serve as a cathode for the drift region.

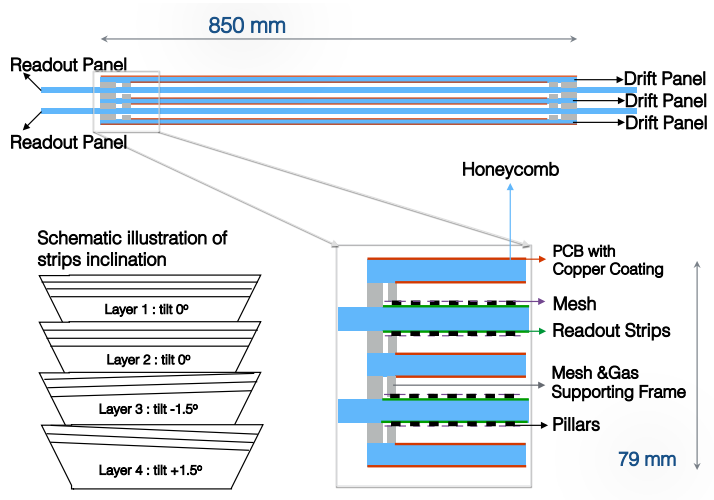

Figure 1: Layout of the detector quadruplet; it consists of three drift and two readout panels $(11.6 \mathrm{~mm}$ thick each), and four $5 \mathrm{~mm}$ thick gas gaps. In Layer 1 and 2 the readout strips are parallel to the trapezoid base while in Layer 3 and 4 the strips are inclined by $\pm 1.5^{\circ}$.

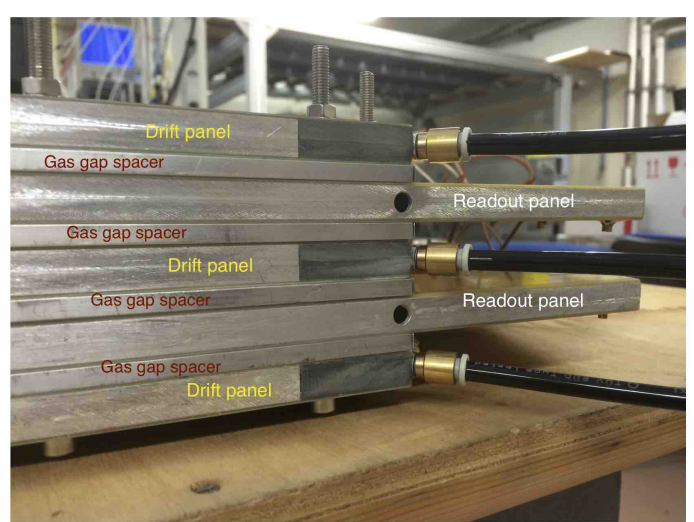

Figure 2: Photo of one corner of the Micromegas quadruplet showing the five panels and the gas gap spacers in between. 

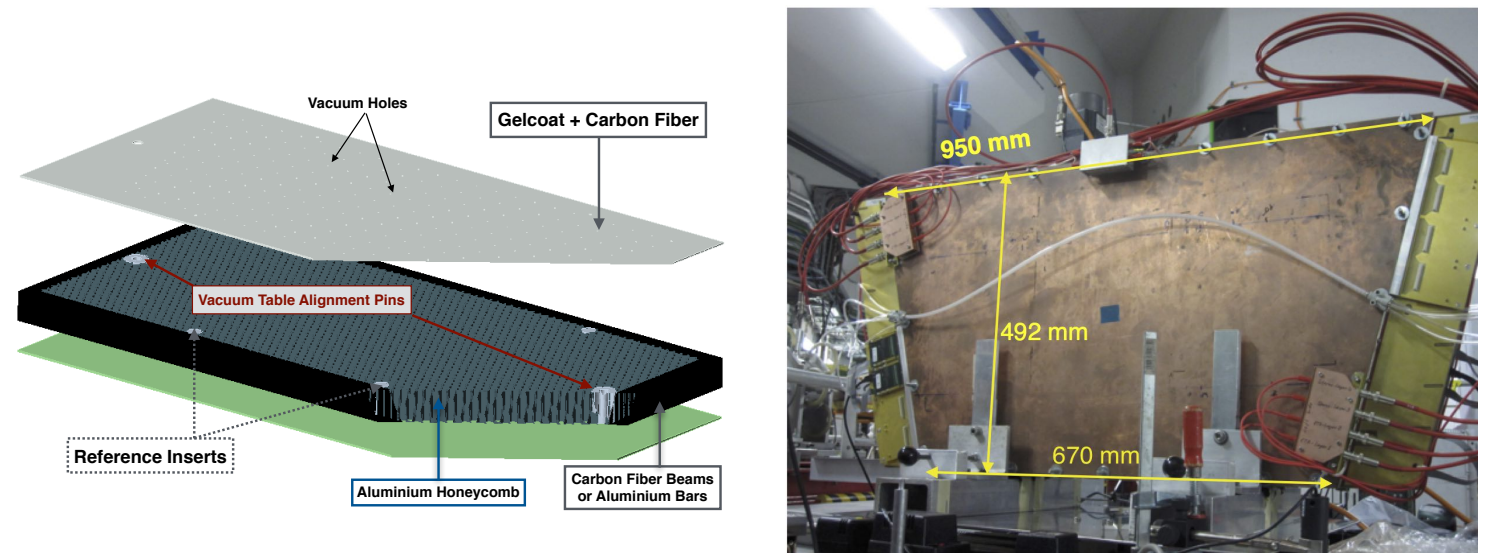

Figure 3: Schematic view of the vacuum table (left) and photo of the one final assembled detector quadruplet equipped with electronics. (right)

\section{Detector Construction}

High planarity of the drift and the readout panels is needed in order to reach the required detector performance, otherwise the particle position can be misconstructed worsen the spatial resolution measurement. For this reason, two vacuum tables were built to construct the panels. The drift and the readout panels were glued as a sandwich structure with aluminum honeycomb in-between as supporting structure and aluminum profile surrounded. Details of the construction are described in [1], [2], hence a brief summary is given in the following.

The vacuum tables are made of $60 \mathrm{~mm}$ perforated aluminium honeycomb, to provide the required stiffness of the table-structure, surrounded by two $3 \mathrm{~mm}$ thick skins of carbon fiber ( Fig. 3 (left)). One of the table surfaces is coated by a $0.5 \mathrm{~mm}$ thick gel-coat layer. The carbon fiber skin together with the gel-coat layer have been prepared and cured on a high-precision granite table transferring the granite table flatness to the vacuum table. The vacuum holes on the tables

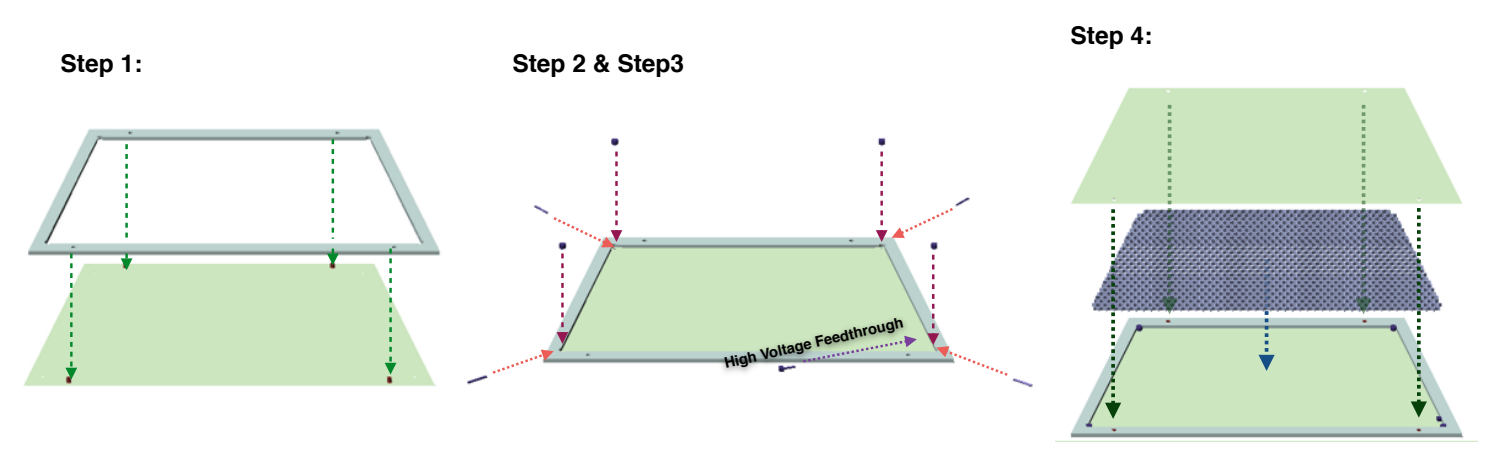

Figure 4: Schematic illustration of the drift panel construction. 


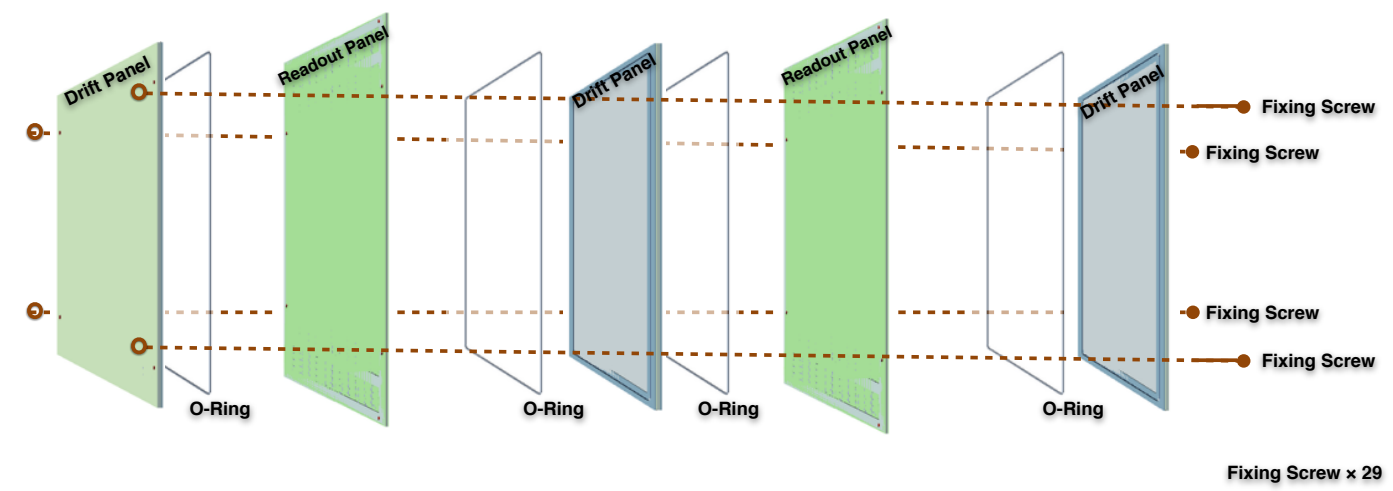

Figure 5: Schematic illustration of the panel assembly procedure. The assembly of the two detector quadruplet was done vertically.

are drilled with a diameter of $1 \mathrm{~mm}$ with regular pattern within the same trapezoidal shape of drift panels.

The panel construction is illustrated in Fig. 4 and described in the following. It is to a large extend identical for the drift and readout panels. In a first step, the aluminium frame is glued to the FR4 skin of the panel. Before the aluminium honeycomb is glued in the space surrounded by the frame, gas connectors and one high voltage feedthrough are implemented to the frame of drift PCB (not for readout PCB). Then the second PCB is glued on the top of the frame and honeycomb structure to close the drift panel. The mesh frame and the gas gap frame together with two gas tubes are glued in addition on the top of the drift panels. In a last step, a uniformly stretched mesh is assembled on the drift panel structure. The assembly sequence of the final detector quadruplet is illustrated in Fig. 5. The gas tightness is ensured by four O-rings, which are inserted between the drift and readout panels around the mesh frames. The distance between the panels is defined by special gas gap spacers, consisting of four $5 \mathrm{~mm}$ thick precision-machined bars with assembly holes. One final assembled detector quadruplet is shown in the right of Fig. 3.

\section{Basic Performance Studies}

The two detector quadruplets have been tested under cosmic rays and in the electron test-beam of MAMI facility in Mainz, Germany.

Fig. 6 left, shows the efficiency of the voltage applied to the resistive strips that defines the correct working point at which the detector should be operated to guarantee good performance. An efficiency of $99 \%$ at the plateau is reached by all the layers. Fig. 6 right, shows in addition, the two-dimensional reconstruction efficiency for one of the quadruplet layers at $580 \mathrm{~V}$.

By increasing the amplification voltage, the saturation probability, i.e. the probability that the charge induced by an incident particle at a given strip is outside the available dynamic range of the readout electronics, rises. This dependence of the saturation probability on amplification voltages is shown in Figure 7. While the saturation probability reaches up to $60 \%$ for the 1 st layer when the highest amplification voltage is applied, it is significantly smaller for the $3 \mathrm{rd}$ and 4 th layer, where a 

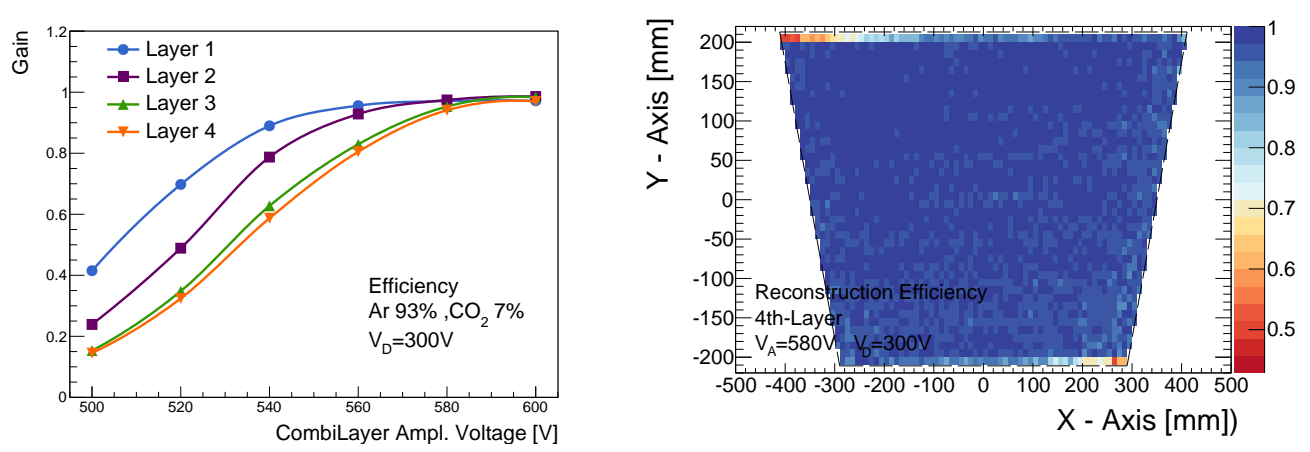

Figure 6: Layer efficiency vs. the amplification voltage (left) and two-dimensional cluster reconstruction efficiency of one layer at $V_{\mathrm{A}}=580 \mathrm{~V}$ (right)

maximal saturation probability of $10 \%$ is observed. The reason for the differences in the saturation probabilities among the layers is their different gain factor. This leads to imprecise position determinations in the saturation regime and hence the maximal saturation probability should be kept low.

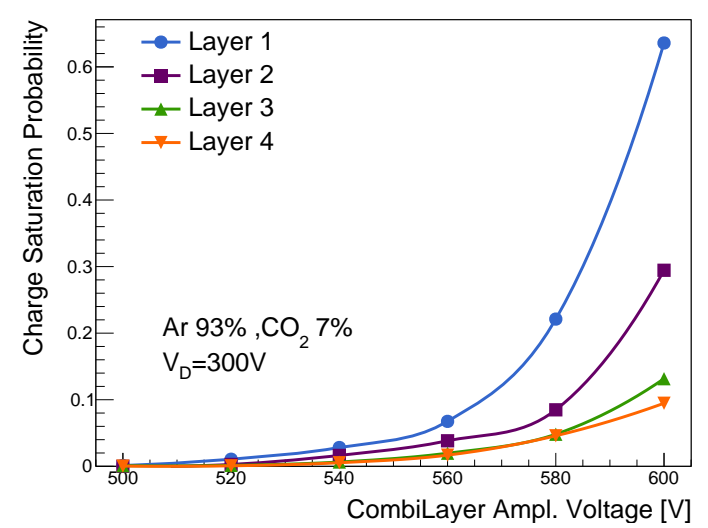

Figure 7: Readout saturation probability vs. the amplification voltage for all layers.

The spatial resolution of the prototype chamber was measured in a $855 \mathrm{MeV}$ electron beam at the MAMI accelerator at the University of Mainz. Fig. 8 shows a resolution of $\sim 100 \mu \mathrm{m}$ for the precision coordinate and $\sim 2.5 \mathrm{~mm}$ for the second coordinate [1]. It should be noted, that multiple scattering effects have not yet been included in this study.

\section{Conclusion}

The construction of two Micromegas quadruplet detectors, in view of the ATLAS muon upgrade, has been described. Each detector has an active area of $0.3 \mathrm{~m}^{2}$ with four layers of onedimensional Micromegas readout structure. Difficulties during the construction of the first detector, have been addressed and improved for the second one. 

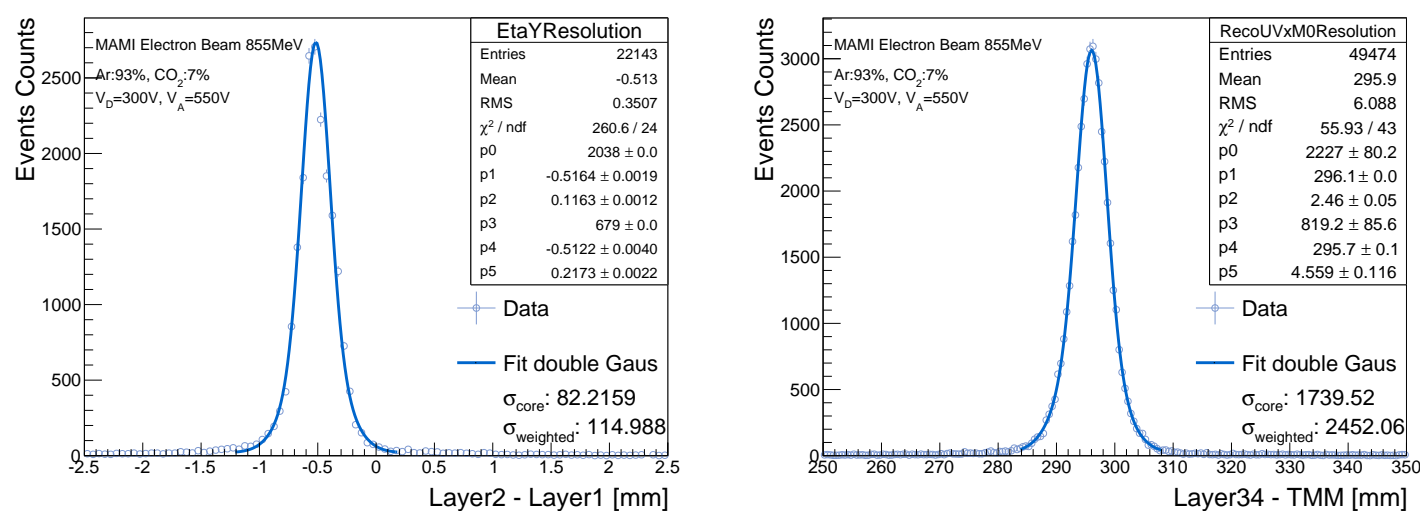

Figure 8: Difference of the reconstructed cluster position of the first and second layer (left) and difference of the y-coordinate position, reconstructed with the 3rd and 4th layer of the MMSW chamber and the reference chamber for test beam events (right).

First performance measurements based on cosmic rays and test beam measurements at the MAMI accelerator have been presented. The response of the detector for different amplification voltages has been studied. An average signal reconstruction efficiency of $99 \%$ per layer was found. The intrinsic spatial resolution was determined in an electron beam of $855 \mathrm{MeV}$ to be better than $100 \mu \mathrm{m}$. A full paper on this project is currently under preparation.

\section{References}

[1] T.-H. Lin, A. Duedder, M. Schott, C. Valderanis, et al. Signal Characteristics of a Resistive-Strip Micromegas Detector with an Integrated Two-Dimensional Readout, Nucl.Instrum.Meth. A767 (2014) 281-288 [arXiv 1406.6871].

[2] M. Bianco, P. Iengo, et al. Construction of a large-size four plane micromegas detector, PoS (TIPP2014) 058

[3] T. Alexopoulosa, C. Amelungb, G. Iakovidis, et al. Micromegas Detectors and Stereo Information, ATLAS Note. (ATL-COM-MUON-2014-047)

[4] A. Duedder, T.-H. Lin, M. Schott, C. Valderanis, et al. Construction of two large-size four-plane micromegas detectors, to be submitted to Nucl.Instrum.Meth. 